\title{
Dirac theory from a field theoretic point of view
}

\author{
Bertfried Fauser \\ Institut für Theoretische Physik \\ Auf der Morgenstelle 14 \\ 72072 Tübingen Germany \\ Electronic mail: Bertfried.Fauser@uni-tuebingen.de
}

May 26, 1996

\begin{abstract}
Several complications arise in quantum field theory because of the infinite many degrees of freedom. However, the distinction between one-particle and many-particle effects - mainly induced by the vacuum - is not clear up to now. A field theoretic picture of the one-particle Dirac theory is developed in order to explore such questions. Main emphasis is laid on the injection of Grassmann's algebra into the endomorphism Clifford algebra built over it. The obtained "field theoretic" functional equation behaves in a very unusual way. New methods to handle Dirac and QFT are given.
\end{abstract}

PACS: 03.65P, 02.10Sp, 11.10 03.75-m

\section{Introduction}

The development of atomic physics was done in several steps, which were adopted to fit the measurements of the contemporary experiments. Thus from Bohr, BohrSommerfeld to Pauli and Dirac [1, 2, 3, 4. there is an increasing accuracy in analogy to the development of a high precise art doing spectroscopic experiments. Beside several improvements concerning additional interactions, there was a need of new concepts also. The discovery of spin and anti-particles was not only a minor change in theory, it was the birth of new concepts in atomic physics. The fact, that the Dirac theory was able to calculate the spectrum of the Hydrogen atom very well can not hide two major drawbacks further present in this theory.

First, the Dirac theory accounts only for the anomalous g-factor 2 and not for the small deviation $(g-2)=\alpha / \pi+\mathcal{O}\left(\alpha^{2}\right)$, which therefore was calculated in quantum electrodynamics (QED), by means of perturbation theory.

Second, despite of the outstanding triumph to predict correctly the appearance of antiparticles, which were not even known in 1928, the mere fact of their introduction spoils the theory to be a one-particle theory by Dirac-sea issues.

Furthermore, one obtains by introducing a QFT vacuum, which exhibits stochastic fluctuations, the Casimir effect. One calculates therewith a force in the empty space of a cavity due to "vacuum polarisation".

The problems seem to be cured in the most accurate present day theory of physics, namely QED. But the necessary(?) field quantization of the gauge field and the so called "second quantization" of the fermion field are another revolutionary development of the theory. There is no commonly accepted way back from QED to Dirac theory.

Now, present day developments in Clifford algebra allow a much more profound reexamination of that important connection. The new calculations, done by Krüger 
[5], with surprising results, show, that something may have been overlooked in the development of Dirac's theory. An exiting evolutionary generalization was promoted by Daviau [6]. Originated in a thoughtful study of the algebraic properties of Dirac's equation, he obtained a non-linear Dirac equation, a special case of Lochak's monopole equation [7], which is called Dirac-Daviau equation. This equation exhibits several new and useful properties 88. Daviau was able to show, that his non-linear equation provides for the Hydrogen atom a nearly analogous spectrum as the linear one, with the additional feature, that the small correction may account for the Lamb separation of otherwise degenerate energy levels.

Now, to turn the way around, one may argue, that it should be possible to find a way back from QED to Dirac's or Daviau's theory. Since it is possible to formulate QED in terms of Clifford algebras [9], this opens a new possibility to connect the two adjacent theories. Moreover, we hope to understand in this way how to manage the distinction of one-particle and multi-particle effects. This is nothing else, as to find an alternative explanation of the so called "vacuum fluctuations". As a first step, we try to find a formulation of the Dirac equation, which exhibits as much as possible the field theoretic features without leaving the one-particle picture.

\section{Clifford algebra as endomorphism algebra of a certain Grassmann algebra}

Definition: The Clifford algebra $C L(\mathcal{V}, \mathcal{Q})$ of the pair $(\mathcal{V}, \mathcal{Q})$ of a linear (vector) space $\mathcal{V}$ and a quadratic form $\mathcal{Q}$ is the universal algebra obtained by the linear Clifford map $\gamma$ satisfying

$$
\begin{aligned}
\gamma: \mathcal{V} \longrightarrow & C L(\mathcal{V}, \mathcal{Q}) \\
\mathcal{V} \ni x \longrightarrow & \gamma_{x} \\
& \gamma_{x} \gamma_{x}=\gamma_{x}^{2}=\mathcal{Q}(x) \\
& C L(\mathcal{V}, \mathcal{Q})=<\operatorname{Id}, \gamma_{x_{i}}, \gamma_{x_{i}} \gamma_{x_{j}, i<j}, \ldots>\text { with } \mathcal{V}=<x_{i}>
\end{aligned}
$$

One might say, that a Clifford algebra is the algebra compatible to a quadratic form. Thereby, every Clifford algebra is related to the geometry induced by the quadratic form $\mathcal{Q}$. In the sequel, we will deal exclusively with non-degenerate quadratic forms. The linear (vector) space $\mathcal{V}$ is assumed to be build over the fields $\mathbb{R}$ or $\mathbb{C}$, denoted by $\mathbb{K}$.

The usual way to introduce the Clifford algebra is to polarize the above Clifford

map $\gamma: \mathcal{V} \rightarrow C L$, to obtain the usual commutation relations. Therefore one has the following

Definition: The polar form $B_{p}$ is the symmetric bilinear form related to $\mathcal{Q}$ by

$$
\begin{aligned}
B_{p}: \mathcal{V} \times \mathcal{V} & \longrightarrow K \\
2 B_{P}(x, y) & =\mathcal{Q}(x+y)-\mathcal{Q}(x)-\mathcal{Q}(y) .
\end{aligned}
$$

The factor 2 was introduced for convenience and causes no problems, because our field $\mathbb{K}$ is of $\operatorname{char} \neq 2(\operatorname{char} \mathbb{K}=0)$.

With a $\mathcal{Q}\left(B_{p}\right)$ orthonormal set of generators $\left\{e_{i}\right\}$, one obtains from (1) the usual commutator relations

$$
\gamma_{e_{i}} \gamma_{e_{j}}+\gamma_{e_{j}} \gamma_{e_{i}}=2 B_{p}\left(e_{i}, e_{j}\right)=2 \operatorname{diag}(+1, \ldots,-1) .
$$

A widely used identification is, to drop the distinction between the linear (vector) space $\mathcal{V}$ and its image $\mathcal{V}(p, q)$ or $\mathcal{V}(n)$ inside the Clifford algebra. We use the term vector space to denote $\mathcal{V}(p, q)$ in $C L$ and linear space for $\mathcal{V}$. Note, that one can 
not speak of a signature without looking at the pair $(\mathcal{V}, \mathcal{Q})$ over $\mathbb{R}$, which is by the universality property essential equivalent to speak about the Clifford algebra $C L(\mathcal{V}, \mathcal{Q}) \equiv C L(p, q)$. The same identification is done with the field $\mathbb{K}$ itself, identifying $\mathbb{K}$ with $\gamma_{\mathbb{K}} \subset C L(\mathcal{V}, \mathcal{Q})$.

A Clifford algebra possesses two important involutions, which are usually given on the set of orthonormalized generators and afterwards on the whole algebra by linearity.

Definition: The main-involution is given by

$$
\begin{aligned}
& : C L \longrightarrow C L \\
& \quad \gamma_{e_{i}} \rightarrow \hat{\gamma}_{e_{i}}=-\gamma_{e_{i}},
\end{aligned}
$$

while the main-anti-automorphism (involution), also called reversion, is given by

$$
\begin{array}{ll}
: & C L \longrightarrow C L \\
& \gamma_{e_{i}} \rightarrow \gamma^{\sim} e_{i}=\gamma_{e_{i}} \\
& \gamma_{e_{i}} \gamma_{e_{j}} \rightarrow\left(\gamma_{e_{i}} \gamma_{e_{j}}\right)^{\sim}=\gamma_{e_{j}} \gamma_{e_{i}}{ }^{\sim}=\gamma_{e_{j}} \gamma_{e_{i}}, \quad \text { etc. }
\end{array}
$$

We define the conjugation ${ }^{-}$as $^{-}:={ }^{\sim} 0^{\wedge}={ }^{\wedge} 0^{\sim}$.

It will become clear later, that a wedge product often introduced by many authors depends on the bilinear form of a Clifford algebra including a possible non-symmetric part. The $\mathbb{Z}_{2}$-grading of a Clifford algebra is obtained from the main-involution, $C L=C L^{+} \oplus C L^{-}$, and thus is not $B_{p}$ or $B$ dependent.

Now, in employing this identification, we have done much more. Knowing a bilinear form $B_{p}$ on $\mathcal{V}$, is equivalent to know an adjoint map from $\mathcal{V}$ to $\mathcal{V}^{*}$, the space of linear forms of $\mathcal{V}$, which itself is a linear (vector) space.

Definition: The adjoint map $*_{B_{p}}$ related to the polar bilinearform $B_{p}$ of $\mathcal{Q}$ is given by

$$
\begin{aligned}
*_{B}: \mathcal{V} \longrightarrow & \mathcal{V}^{*} \\
& \mathcal{V}^{*}(p, q) \ni x^{*_{B}}:=B(x, \cdot) \\
& x^{*_{B}}(y)=B(x, y) . \square
\end{aligned}
$$

Now, it is well known, that there is no canonical dual isomorphism, which relates $\mathcal{V}$ and $\mathcal{V}^{*}$ as linear spaces. But, as we have seen, the Clifford algebra provides us with such a dual isomorphism by its construction. Thus we are dealing with the pair $\left(C L(\mathcal{V}, \mathcal{Q}), *_{B}\right)$, the Clifford algebra and the dual isomorphism.

As we are studying the finite dimensional case, no difficulties should occur, but it is exactly this step, which is crucial in field theory. The dual space $\mathcal{V}^{*}$ of an infinite dimensional space $\mathcal{V}$ may be of larger cardinality as $\mathcal{V}$. In this case, we are not able to identify $\mathcal{V}$ with $\mathcal{V}^{* *}$ in a canonical way.

But have we done the thing right already in the finite theory?

Because the Clifford algebra is the universal algebra of a linear (vector) space and a quadratic form, we have to look, if every non-singular bilinear form, which gives us a dual isomorphism is obtained in this way. The answer is the following Proposition: The symmetric part of a non-singular bilinear form determines the corresponding quadratic form uniquely.

Thus the Projection from the space of bilinear forms onto the space of quadratic forms is not surjectiv,

$$
\frac{\text { bilinear forms }}{\text { alternating forms }} \cong \text { quadratic forms. }
$$

Now, not being able to construct a unique bilinear form from a quadratic form, we are still not able to construct a dual isomorphism. 
Since quantum mechanics, as quantum field theory in a much more elaborated way, is based on states and duality in the sense of an adjoint, we are not jet able to study quantum mechanics with Clifford algebras. But on the other hand, simple one-particle quantum mechanics does fix the choice immediately and so also omits this freedom.

In the Hestenes formulation of Dirac's theory [10] we have simply

$$
\begin{array}{lll}
\mid \psi> & \overrightarrow{D H} & \Psi \in C L^{+} \\
<\psi \mid & \stackrel{D H}{\Psi} & \tilde{\Psi} \in C L^{+} .
\end{array}
$$

Thus the Dirac adjoint is represented by the reversion, which was defined on the orthonormalized generators of $C L(\mathcal{V}, \mathcal{Q})$.

To be able to give a more elaborate answer to the above question, we will construct the Clifford algebra from the Grassmann algebra by Chevalley deformation. This process directly relates an arbitrary bilinear form $B$ to the pair $\left(C L(\mathcal{V}, \mathcal{Q}), *_{B}\right)$ in a unique way.

Definition: The Grassmann algebra $\Lambda[\mathcal{V}]$ of the linear (vector) space $\mathcal{V}$ is the universal algebra obtained by the linear injection $j$ with the properties

$$
\begin{aligned}
& j: \mathcal{V} \longrightarrow \Lambda[\mathcal{V}] \\
& \mathcal{V} \ni x \longrightarrow j_{x} \\
& j_{x} \wedge j_{x}=j_{x}^{2}=0 .
\end{aligned}
$$

The product in $\Lambda[\mathcal{V}]$ is often denoted by the $\wedge$ (wedge product).

Remark: Because of the fact, that the wedge product, called "combinatorial product (Kombinatorisches Produkt)" by Grassmann 11, describes a disjoint union one should use the vee product $\vee$ instead.

The Grassmann algebra of a finite dimensional linear (vector) space $\mathcal{V}, \operatorname{dim} \mathcal{V}=$ $n$, is a $\mathbb{Z}_{n}$-graded finite (unital, associative $\mathbb{K}^{-}$) algebra of dimension $2^{n}$. Each element of $\Lambda[\mathcal{V}]$ can be decomposed into parts of homogeneous degree. Thus

$$
\begin{aligned}
\Lambda[\mathcal{V}] & =\oplus_{r=0}^{n} \Lambda^{r}[\mathcal{V}] \\
\operatorname{dim}: 2^{n} & =\sum_{r=0}^{n}\left(\begin{array}{l}
n \\
r
\end{array}\right) .
\end{aligned}
$$

Given a set $\left\{x_{i}\right\}$, which generates $\mathcal{V}$ as linear space, every element $X$ in $\Lambda[\mathcal{V}]$ can be written as (summation convention employed)

$$
\begin{aligned}
X= & \alpha_{0} \operatorname{Id}+\alpha_{i} j_{x_{i}}+\alpha_{i j} j_{x_{i}} \wedge j_{x_{j}, i<j}+\ldots \\
& \left\{\alpha_{0}, \alpha_{i}, \ldots\right\} \in \mathbb{K} .
\end{aligned}
$$

Obviously, $\Lambda[\mathcal{V}]$ can also be decomposed into even and odd parts by the maininvolution

$$
\begin{aligned}
& : \mathcal{V} \longrightarrow-\mathcal{V} \\
& \hat{j}_{x_{i}}=-j_{x_{i}} \quad \forall j_{x_{i}} \quad \in \mathcal{V} \\
& \Lambda[\mathcal{V}]=\Lambda^{+}[\mathcal{V}] \oplus \Lambda^{-}[\mathcal{V}]
\end{aligned}
$$

To reobtain the Clifford algebra, we introduce the dual space of linear forms $\mathcal{V}^{*}$ of the linear (vector) space $\mathcal{V}$. With the same generating set $\left\{x_{i}\right\}$ of $\mathcal{V}$ we have the Definition: The dual base in $\mathcal{V}^{*}$ is given by the co-vectors $\partial^{x_{i}}$ subjected to the conditions

$$
\partial^{x_{i}} j_{x_{j}}=\delta_{j}^{i} \cdot \square
$$


In this way the action of every co-vector element $a \in \mathcal{V}^{*}$ on the vector elements $x \in \mathcal{V}$ is given. But to ensure the action of the co-vectors on multi-vectors out of $\Lambda[\mathcal{V}]$, we have to require, that the $\partial^{x}$ are anti-derivations of degree -1 . In addition, we have to define how to apply repeated derivations and the action of multi-covectors. This leads to the well known properties of $\partial^{x}$ on the generators, which by linearity are defined on the whole algebra [12]

$$
\begin{aligned}
& \partial^{\alpha x_{i}+\beta x_{j}}=\alpha \partial^{x_{i}}+\beta \partial^{x_{j}} \\
& \partial^{x_{i}}\left(j_{x_{j}}\right)=\delta_{j}^{i} \\
& \partial^{x_{i}} j_{x_{j_{1}}} \wedge \ldots \wedge j_{x_{j_{n}}}= \\
& \text { linearity } \\
& \text { def. of co-vectors, } x_{i} \in \mathcal{V} \\
& \sum_{r=1}^{n}(-)^{r+1} \delta_{j_{r}}^{i} j_{x_{j_{1}}} \wedge \ldots \wedge j_{x_{j_{r-1}}} \wedge j_{x_{j_{r+1}}} \wedge \ldots \wedge j_{x_{j_{n}}} \\
& \partial^{x_{i_{1}} \wedge \ldots \wedge x_{i_{r}}} j_{x_{j_{1}}} \wedge \ldots \wedge j_{x_{j_{n}}}=\quad \text { left module structure } \\
& \partial^{x_{i_{1}}}\left(\ldots\left(\partial^{x_{i_{r}}}\left(j_{x_{j_{1}}} \wedge \ldots \wedge j_{x_{j_{n}}}\right)\right) \ldots\right) \quad\left(=\operatorname{det} \partial^{x_{i}} j_{x_{j}} \text { if } \# x_{i}=\# x_{j}\right)
\end{aligned}
$$

Therefore, we are able to build up from the dual space $\mathcal{V}^{*}$ also a Grassmann algebra, acting on $\Lambda[\mathcal{V}]$ in a multilinear way. But one has to care not to confuse $\Lambda\left[\mathcal{V}^{*}\right]=$ $\Lambda\left[<\partial^{x_{i}}>\right]$ with $[\Lambda[\mathcal{V}]]^{*}$.

If we look at the endomorphisms of $\Lambda[\mathcal{V}]$, we have the following

Theorem: (Greub [13, 14])

$$
\begin{aligned}
\operatorname{End}(\Lambda[\mathcal{V}]) \cong & C L\left(\mathcal{V} \oplus \mathcal{V}^{*}, \delta \perp \delta\right) \cong C L(\mathcal{V} \oplus \mathcal{V}, \delta \perp-\delta) \cong \Lambda[\mathcal{V}] \otimes \Lambda\left[\mathcal{V}^{*}\right] \\
& \delta\left(x_{i}\right)=x_{i} x_{i}=1, \quad \mathcal{V}=<x_{i}>, \quad \mathcal{V}^{*}=<\partial^{x_{i}}>
\end{aligned}
$$

In another language, we may look at the elements of the Grassmann algebra as states and at the elements of the Clifford algebra as operators, acting by left multiplication on states.

To make this connection more explicit, we give the

\section{Definition:}

$$
\begin{aligned}
\gamma^{x_{i}} & :=\sum_{j} \delta^{x_{i} x_{j}} j_{x_{j}}+\partial^{x_{i}} \\
\gamma^{* x_{i}} & :=\sum_{j} \delta^{x_{i} x_{j}} j_{x_{j}}-\partial^{x_{i}} \square
\end{aligned}
$$

From this we can easily calculate the commutation relations

$$
\begin{aligned}
\gamma^{x_{i}} \gamma^{x_{j}}+\gamma^{x_{j}} \gamma^{x_{i}} & =2 \delta^{i j} \\
\gamma^{* x_{i}} \gamma^{* x_{j}}+\gamma^{* x_{j}} \gamma^{* x_{i}} & =-2 \delta^{i j} \\
\gamma^{x_{i}} \gamma^{* x_{j}}+\gamma^{* x_{j}} \gamma^{x_{i}} & =0
\end{aligned}
$$

Of course, this construction is a cover of $\operatorname{End}(\Lambda[\mathcal{V}])$, which is generated by the $\gamma^{x_{i}}$ and $\gamma^{* x_{i}}$. Nevertheless, by dimensional arguments, we have $\operatorname{dim} \Lambda[\mathcal{V}]=2^{n}$ and $\operatorname{dim} \operatorname{End}[\Lambda[\mathcal{V}]]=2^{2^{n}}$ and need therefore both kinds of $\gamma$ 's to establish the connection between the $\gamma$ and the $j, \partial$ picture. We may invert (17), which is possible for every non-degenerate symmetric bilinear form (non-degenerate $\mathcal{Q}$ ) and obtain (s.c. employed)

$$
\begin{aligned}
j_{x_{i}} & =\frac{1}{2} \delta_{x_{i} x_{j}}\left(\gamma^{x_{j}}+\gamma^{* x_{j}}\right) \\
\partial^{x_{i}} & =\frac{1}{2}\left(\gamma^{x_{i}}-\gamma^{* x_{i}}\right)
\end{aligned}
$$


If we introduce the involution $*$ by

$$
\begin{aligned}
*: & C L\left(\mathcal{V} \oplus \mathcal{V}^{*}, \delta \perp \delta\right) \longrightarrow C L\left(\mathcal{V} \oplus \mathcal{V}^{*}, \delta \perp \delta\right) \\
& \gamma \rightarrow \gamma^{*} \\
& \gamma^{*} \rightarrow \gamma
\end{aligned}
$$

we obtain $\Lambda[\mathcal{V}]$ as the space, which is stabilized by this $*$-involution.

Because of the

Theorem: (Chevalley, 15])

$$
C L(\mathcal{V},-\mathcal{Q}) \cong C L(\mathcal{V}, \mathcal{Q})^{o p}
$$

where $C L^{o p}$ is the algebra with reversed products, we can identify the $\gamma^{* x_{i}}$ as a $\gamma^{x_{i}}$ acting from the right. Set $u=\gamma^{x_{1}} \ldots \gamma^{x_{n}}$, then we have (see also [16])

$$
\begin{aligned}
* \quad: \quad & C l \longrightarrow C l^{o p} \\
& *\left(\gamma^{x_{k}} u\right)=\hat{u} \gamma^{* x_{k}}
\end{aligned}
$$

Thus, due to the necessity of working with (vector) states in quantum mechanics, we have to take care to simulate the right action of $\gamma^{x_{i}}$ by the left action of $\gamma^{* x_{i}}$. The involution $*$ may be looked at as a sort of transposition or (Hermite) adjoint.

Now, the same construction is possible for an arbitrary non-degenerate not necessary symmetric bilinear form $B$, with symmetric part $A$ and anti-symmetric part $F$. The matrices of these bilinear forms on the generating set $\left\{x_{i}\right\}$ will be denoted by $\left[B^{i j}\right]=\left[B\left(x_{i}, x_{j}\right)\right]$. We end up with the following formulas (s.c. employed)

$$
\begin{aligned}
B^{o p}(x, y) & =-B(y, x)=-A+F \\
\gamma^{x_{i}} & :=\left[B^{i j}\right] j_{x_{j}}+\partial^{x_{i}}=\left[A^{i j}\right] j_{x_{j}}+\left[F^{i j}\right] j_{x_{j}}+\partial^{x_{i}} \\
\gamma^{* x_{i}} & :=\left[B^{o p}{ }^{i j}\right] j_{x_{j}}-\partial^{x_{i}}=-\left[A^{i, j}\right] j_{x_{j}}+\left[F^{i j}\right] j_{x_{j}}-\partial^{x_{i}} \\
{\left[A^{i j}\right] } & \equiv\left[A_{i j}^{-1}\right] \quad(\text { exists by assumption }) \\
j_{x_{i}} & :=\frac{1}{2}\left[A_{i j}\right]\left(\gamma^{x_{j}}+\gamma^{* x_{j}}\right) \\
\partial^{x_{i}} & :=\frac{1}{2}\left(\gamma^{x_{i}}-\gamma^{* x_{i}}\right)-\frac{1}{2}\left[F^{i j}\right]\left[A_{j k}\right]\left(\gamma^{x_{k}}+\gamma^{* x_{k}}\right) .
\end{aligned}
$$

We drop in further formulas the brackets [.]. It is obvious, that this sort of new $\gamma$ 's are subjected to new commutation relations

$$
\begin{array}{rlr}
\gamma^{x_{i}} \gamma^{x_{j}}+\gamma^{x_{j}} \gamma^{x_{i}} & =2 A^{i j} \\
\gamma^{* x_{i}} \gamma^{* x_{j}}+\gamma^{* x_{j}} \gamma^{* x_{i}} & =-2 A^{i j} \\
\gamma^{x_{i}} \gamma^{* x_{j}}+\gamma^{* x_{j}} \gamma^{x_{i}} & =0,
\end{array}
$$

and that the involution $*$ does no longer stabilize the space spanned by the $j$-sources. Hence, this transformation can no longer be identified by a matrix transposition. Furthermore, the alternating part $F$ is completely absent in this commutators.

We have to find a meaning of the norms, which are important to describe "scalar products". The usual way [17, 18] is, to study the fields (double fields) real Clifford algebras are built over. With the notations ${ }^{2} \mathbb{R}=\mathbb{R} \oplus \mathbb{R}$ and $\mathbb{R}(2)=$ Mat $_{2 \times 2}(\mathbb{R})$, we have the relevant left modules built over

$$
M:=\left\{\mathbb{R}, \mathbb{C}, \mathbb{H},{ }^{2} \mathbb{R},{ }^{2} \mathbb{H}\right\}
$$

These modules are the spaces of (double) spinors, only one of them equals $\mathbb{C}$. 
We may utilize two different anti-automorphisms to build up scalar products.

\section{Definition:}

$$
\alpha_{\varepsilon}:=\left\{\begin{array}{cll}
\sim & \Leftrightarrow & \varepsilon=+1 \\
-=^{\sim} \circ^{\wedge} & \Leftrightarrow & \varepsilon=-1 \square
\end{array}\right.
$$

We have thus $\alpha_{\varepsilon}(\mathcal{V}(p, q))=\varepsilon \mathcal{V}(p, q)$ etc. With help of this map we may build a form $\Phi_{\varepsilon}$ by

$$
\begin{aligned}
\Phi_{\varepsilon}: \quad & C L(\mathcal{V}, \mathcal{Q}) \times C L(\mathcal{V}, \mathcal{Q}) \longrightarrow C L(\mathcal{V}, \mathcal{Q}) \\
& \Phi_{\varepsilon}\left(r_{1}, r_{2}\right)=\alpha_{\varepsilon}\left(r_{1}\right) r_{2} .
\end{aligned}
$$

For any $g \in C L(\mathcal{V}, \mathcal{Q})$, we have the left $C L(\mathcal{V}, \mathcal{Q})$-modul homomorphism

$$
\begin{aligned}
g: \quad & C L(\mathcal{V}, \mathcal{Q}) \longrightarrow C L(\mathcal{V}, \mathcal{Q}) \\
& g(r)=g r,
\end{aligned}
$$

and the adjoint w.r.t. $\Phi_{\varepsilon}$ is given by $\alpha_{\varepsilon}(g)$. In this way, the form $\Phi_{\varepsilon}$ induces the map

$$
\begin{aligned}
\mathrm{N}_{\varepsilon} \quad: \quad & C L(\mathcal{V}, \mathcal{Q}) \longrightarrow C L(\mathcal{V}, \mathcal{Q}) \\
& \mathrm{N}_{\varepsilon}(r)=\alpha_{\varepsilon}(r) r=\Phi_{\varepsilon}(r, r) \\
& \mathrm{N}_{\varepsilon}(v)=\varepsilon v^{2}=\varepsilon \mathcal{Q}(v), \quad v \in \mathcal{V}(p, q) .
\end{aligned}
$$

The invariance group of $\Phi_{\varepsilon}$ (under multiplication) is given by

$$
\operatorname{Inv}_{\varepsilon}(p, q)=\left\{g \in C L(\mathcal{V}, \mathcal{Q}) \mid \mathrm{N}_{\varepsilon}(g)=1\right\} .
$$

Lounesto has shown, that there exists always a form equivalent (isomorphic) to $\Phi_{\varepsilon}$, such that the restriction of $\mathrm{N}_{\varepsilon}$ to (double)- spinors is $M$-valued [19]. The group

of automorphisms, which preserve the "scalar product" $\Phi_{\varepsilon}$ is thus isomorphic to $\operatorname{Inv} v_{\varepsilon}(p, q)$.

Definition: (Lipschitz group) Let $C L^{\times}(\mathcal{V}, \mathcal{Q})$ denote the units in $C L(\mathcal{V}, \mathcal{Q})$ w.r.t. $\Phi_{\varepsilon}$. The Lipschitz group $\Gamma(p, q)$ is given as

$$
\Gamma(p, q):=\left\{r \in C L^{\times}(\mathcal{V}, \mathcal{Q}) \mid r \mathcal{V}(p, q) \hat{r}^{-1} \subset \mathcal{V}(p, q)\right\}
$$

For every $r \in \Gamma(p, q)$,

$$
\begin{aligned}
\rho_{r} \quad: \quad & \mathcal{V}(p, q) \longrightarrow \mathcal{V}(p, q) \\
& \rho_{r}(v)=r v \hat{r}^{-1}
\end{aligned}
$$

belongs to $\mathbf{O}(p, q)$. Further we have the important

Proposition: The pseudo-norm $\mathrm{N}_{\varepsilon}$ is $\mathbb{K}$ valued on $\Gamma(p, q)$.

Because one can write every element $r$ of $\Gamma(p, q)$ as $r=v_{1} \ldots v_{n}$, with $v_{i} \in$ $\mathcal{V}(p, q)$, we obtain

$$
\mathrm{N}_{\varepsilon}=\alpha_{\varepsilon}(r) r=\varepsilon^{n} \mathcal{Q}\left(v_{1}\right) \ldots \mathcal{Q}\left(v_{n}\right)
$$

which is in $\mathbb{K}$.

Thus, our above construction involves an anti-automorphism and thereby induce in general a $M$-valued (degenerate) form on the whole algebra. We have thus to search for "states", which are in $\Gamma(p, q)$ (pure states), to obtain $\mathbb{K}$-valued "expectation values". 
Before we proceed to study Dirac's theory with $j$-sources and Chevalley deformation, we consider the conjugations. From the linear dependence of $\gamma, \gamma^{*}$ from $j, \partial(23)$ we have

$$
\begin{aligned}
\hat{\gamma}^{x_{i}} & =-\left(\gamma^{x_{i}}\right)=-\left(\partial^{x_{i}}+B^{i j} j_{x_{j}}\right) \\
& =(-\partial)^{x_{i}}+B^{i j}(-j)_{x_{j}}
\end{aligned}
$$

and thus

$$
\begin{aligned}
& \hat{\partial}^{x_{i}}=-\partial^{x_{i}} \\
& \hat{j}_{x_{i}}=-j_{x_{i}} .
\end{aligned}
$$

From

$$
\begin{aligned}
\gamma^{x_{k}} \gamma^{x_{i}} & =\partial^{x_{k}} \partial^{x_{i}}+B^{i k}-B^{i l} j_{x_{l}} \partial^{x_{k}}+B^{k j} j_{x_{j}} \partial^{x_{i}}+B^{i j} B^{k l} j_{x_{l}} j_{x_{j}} \\
& =\left(\partial^{x_{i}} \partial^{x_{k}}+B^{i k}-B^{i l} \partial^{x_{k}} j_{x_{l}}+B^{k j} \partial^{x_{i}} j_{x_{j}}+B^{i j} B^{k l} j_{x_{j}} j_{x_{l}}\right)^{\sim} \\
& =\left(\partial^{x_{i}} \partial^{x_{k}}+B^{i k}-B^{i k}+B^{k l} j_{x_{l}} \partial^{x_{i}}+B^{k i}-B^{i l} j_{x_{l}} \partial^{x_{k}}+B^{i j} B^{k l} j_{x_{j}} j_{x_{l}}\right)^{\sim} \\
& =\left(\gamma^{x_{i}} \gamma^{x_{k}}\right)^{\sim},
\end{aligned}
$$

we conclude that ${ }^{\sim}$ reverse products of $j$ and $\partial$. Thus

$$
\left(j_{x_{r_{1}}} \ldots j_{x_{r_{n}}} \partial^{x_{s_{1}}} \ldots \partial^{x_{s_{m}}}\right)^{\sim}=\partial^{x_{s_{m}}} \ldots \partial^{x_{s_{1}}} j_{x_{r_{n}}} \ldots j_{x_{r_{1}}} .
$$

e.g.

$$
\begin{aligned}
\left(j_{x_{i}} \partial^{x_{k}}\right)^{\sim} & =\partial^{x_{k}} j_{x_{i}}=\delta_{i}^{k}-j_{x_{i}} \partial^{x_{k}} \\
\left(j_{x_{i}} \partial^{x_{j}} \partial^{x_{k}}\right)^{\sim} & =\delta_{i}^{j} \partial^{x_{k}}-\delta_{i}^{k} \partial^{x_{j}}+j_{x_{i}} \partial^{x_{k}} \partial^{x_{j}}
\end{aligned}
$$

The star conjugation results in

$$
\begin{aligned}
j_{x_{i}}^{*} & =\left[\frac{1}{2} A_{i j}\left(\gamma^{x_{j}}+\gamma^{* x_{j}}\right)\right]^{*} \\
& =\frac{1}{2} A_{i j}\left(\gamma^{* x_{j}}+\gamma^{x_{j}}\right)=j_{x_{i}} \\
\partial^{* x_{i}} & =\left[\frac{1}{2}\left(\gamma^{x_{i}}-\gamma^{* x_{i}}\right)-\frac{1}{2} F^{i j} A_{j k}\left(\gamma^{x_{k}}+\gamma^{* x_{k}}\right)\right]^{*} \\
& =-\frac{1}{2}\left(\gamma^{x_{i}}-\gamma^{* x_{i}}\right)+\frac{1}{2} F^{i j} A_{j k}\left(\gamma^{x_{k}}+\gamma^{* x_{k}}\right)-F^{i j} A_{j k}\left(\gamma^{x_{k}}+\gamma^{* x_{k}}\right) \\
& =-\partial^{x_{i}}-2 F^{i j} j_{x_{j}} \\
\partial^{* * x_{i}} & =-\partial^{* x_{i}}-2 F^{i j} j_{x_{j}}=\partial^{* x_{i}}+2 F^{i j} j_{x_{j}}-2 F^{i j} j_{x_{j}} \\
& =\partial^{x_{i}}
\end{aligned}
$$

This suggests to introduce the new derivative

$$
\begin{aligned}
\mathrm{d}^{x_{i}} & :=\partial^{x_{i}}+F^{i j} j_{x_{j}} \\
\mathrm{~d}^{* x_{i}} & =-\partial^{x_{i}}-2 F^{i j} j_{x_{j}}+F^{i j} j_{x_{j}}=-\left(\partial^{x_{i}}+F^{i j} j_{x_{j}}\right)=-\mathrm{d}^{x_{i}},
\end{aligned}
$$

which is thus $*$ anti-stable. Of course, exact this was the motivation to introduce normal ordering in QFT [20]. The commutator relations are derived to be

$$
\begin{aligned}
\left\{\mathrm{d}^{x_{i}}, \mathrm{~d}^{x_{j}}\right\} & =0 \\
\left\{\mathrm{~d}^{x_{i}}, j_{x_{j}}\right\} & =\delta_{j}^{i} \\
\left\{j_{x_{i}}, j_{x_{j}}\right\} & =0 .
\end{aligned}
$$


Thus we obtain a new set of co-vectors, subjected to the same commutation relations. But, the star conjugation (anti) stabilizes the $\mathrm{d}, j$ parameterization and not the $\partial, j$ set. One reobtains in the $\mathrm{d}, j$ picture the usual matrix transposition. The substitution $\partial \rightarrow \partial+F j$ is exactly the functional form of normal ordering in QFT, if one asserts $F$ to be the propagator [21, 97.

For defining the adjoint, we combine the reversion and conjugation again into $\alpha_{\varepsilon}$ (26). We obtain the action of $\alpha_{\varepsilon}$ as

$$
\begin{aligned}
\alpha_{\varepsilon}\left(j_{x_{i}}\right) & =\varepsilon j_{x_{i}} \\
\alpha_{\varepsilon}\left(\partial^{x_{i}}\right) & =\varepsilon \partial^{x_{i}} \\
\alpha_{\varepsilon}\left(j_{x_{1}} \ldots j_{x_{n}} \partial^{x_{n+1}} \ldots \partial^{x_{n+r}}\right) & =\alpha_{\varepsilon}\left(\partial^{x_{n+r}}\right) \ldots \alpha_{\varepsilon}\left(\partial^{x_{n+1}}\right) \alpha_{\varepsilon}\left(j_{x_{n}}\right) \ldots \alpha_{\varepsilon}\left(j_{x_{1}}\right) \\
& =\varepsilon^{n+r} \partial^{x_{n+r}} \ldots \partial^{x_{n+1}} j_{x_{n}} \ldots j_{x_{1}} .
\end{aligned}
$$

In low dimensional cases one obtains

$$
\begin{aligned}
\alpha_{\varepsilon}(\mathrm{Id}) & =\mathrm{Id} \\
\alpha_{\varepsilon}\left(j_{x_{i}} \partial^{x_{k}}\right) & =\varepsilon^{2} \partial^{x_{k}} j_{x_{i}}=\delta_{i}^{k}-j_{x_{i}} \partial^{x_{k}} \\
\alpha_{\varepsilon}\left(j_{x_{i}} j_{x_{k}} \partial^{x_{l}}\right) & =\varepsilon^{3} \partial^{x_{l}} j_{x_{k}} j_{x_{i}}=\varepsilon\left(\delta_{k}^{l} j_{x_{i}}-\delta_{i}^{l} j_{x_{k}}-j_{x_{i}} j_{x_{k}} \partial^{x_{l}}\right) \\
& =\left(\delta_{k}^{l}-j_{x_{k}} \partial^{x_{l}}\right) \varepsilon\left(j_{x_{i}}\right) \\
& =\alpha_{\varepsilon}\left(j_{x_{k}} \partial^{x_{l}}\right) \alpha_{\varepsilon}\left(j_{x_{i}}\right) .
\end{aligned}
$$

The derivation structure is thus compatible with the action of the adjoint. Hence, we are able to define a form on "states", which are built of $j$-sources and a projector $\left|0>_{F}<0\right|($ see 58);

$$
\begin{aligned}
\mid X> & =\sum_{i, j, k, \ldots}\left(\alpha_{0} \mathrm{Id}+\alpha_{i} j_{x_{i}}+\ldots\right)\left|0>_{F}<0\right| \\
<X \mid & :=\alpha_{\varepsilon}(\mid X>) \\
\Phi_{\varepsilon}(X, Y) & :=\alpha_{\varepsilon}(\mid X>)|Y>=<X| Y>\in C L(p, q)
\end{aligned}
$$

If we require the $\mid X>$ to be in the Lipschitz group $\Gamma(p, q)$, we obtain a "scalar product" into the center of $C L(p, q)$, which is $M$-valued.

Remark: Up to this state of the development, we are not able to utilize the dual isomorphism $c o \mathcal{V}$,

$$
\begin{aligned}
\operatorname{cov}\left(j_{x_{i}}\right) & =\partial^{x_{i}} \\
\operatorname{cov}\left(\partial^{x_{i}}\right) & =j_{x_{i}},
\end{aligned}
$$

because of the raising and lowering of the involved indices, which could be done by $A, F, B$ or in another way. In a Cartesian picture with $\partial^{i}=\partial_{i}, j^{i}=j_{i}, c o \mathcal{V}$ would lead to a Fock space construction [22].

\section{Dirac theory}

Dirac theory is usually given in terms of matrix representations. For the purpose of reference, we use Bjorken and Drell [23],

$$
\sum_{\beta=1}^{4}\left(\sum_{\mu=0}^{3}\left(i \hbar \gamma_{\alpha \beta}^{\mu} \frac{\partial}{\partial x^{\mu}}+\frac{e}{c} \gamma_{\alpha \beta}^{\mu} A_{\mu}\right)+m c \delta_{\alpha \beta}\right) \psi_{\beta}=0
$$

where $\psi_{\beta}$ is a component of a column spinor, hence $\psi_{\beta}: \mathrm{M}_{1,3} \longrightarrow \mathbb{C}$. The $\gamma$ matrices are elements of $\operatorname{Mat}_{4 \times 4}(\mathbb{C})$. The spinor inner product is given by the Dirac adjoint 
$\bar{\psi}_{\beta}^{D}=\left(\gamma^{0} \psi\right)_{\beta}^{* T}$, where $*$ is complex conjugation and $T$ denotes transposition of the matrix representation. Thus

$$
\sum_{\beta=1}^{4} \bar{\psi}_{\beta}^{D} \psi_{\beta}=\alpha \in \mathbb{R}
$$

is the standard scalar product. The polar bilinear form is just the diagonal matrix $\eta^{\mu \nu}=\operatorname{diag}(1,-1,-1,-1)$, which can be seen from

$$
\sum_{\beta=1}^{4}\left(\gamma_{\alpha \beta}^{\mu} \gamma_{\beta \rho}^{\nu}+\gamma_{\alpha \beta}^{\nu} \gamma_{\beta \rho}^{\mu}\right)=2 \eta^{\mu \nu} \delta_{\alpha \rho}
$$

Now, there are several methods to establish a connection to Clifford algebraic spinors. Since we are interested in "states", we prefer ideal spinors [24], because of their modul structure.

Let us start from an orthonormalized set of generating elements $\left\{e^{\mu}\right\}$, and the bilinear form $B=B\left(e^{\mu}, e^{\nu}\right)=\eta^{\mu \nu}+F^{\mu \nu}$, with an arbitrary function $F^{\mu \nu}$. This is no restriction since $\eta^{\mu \nu}$ is constant, because we are able to diagonalize the nondegenerate symmetric part of the bilinear form, and afterwards normalize it to \pm 1 according to Sylvester's theorem. This results thereby in a change of the alternating part also!

We can built a primitive idempotent element $P$ as

$$
P \equiv P_{11}=\frac{1}{2}\left(\mathrm{Id}+e^{0}\right) \frac{1}{2}\left(\mathrm{Id}+i e^{2} e^{3}\right) .
$$

The appearance of a non-geometric $i$ is due to the fact, that we are interested in a $\operatorname{Mat}_{4 \times 4}(\mathbb{C})$ representation of the Dirac theory, which is quite artificial, since the Dirac algebra $C L_{1,3}(\mathbb{R}) \equiv C L\left(\mathbb{R}^{4}, \eta\right)$ should be represented in $\operatorname{Mat}_{2 \times 2}(\mathbb{H})$. Nevertheless, the throughout appearance of $i \gamma^{2}$ has also physical consequences. One might look at Dirac theory as a theory over $C L_{2,2}\left(\mathbb{R}^{4}\right)$, which is totally null and twistor like. This algebra can be represented in $\operatorname{Mat}_{4 \times 4}(\mathbb{R})$. For example, angular momentum is studied most easily with $l_{ \pm}:=\gamma^{1} \pm i \gamma^{2}, l^{3}:=\gamma^{3}$ and $\gamma^{5}$ is usually defined as $\gamma^{5}:=i\left(\gamma^{0} \ldots \gamma^{3}\right)=\gamma^{0} \gamma^{1}\left(i \gamma^{2}\right) \gamma^{3}$, which reflects our choice.

We define the set

$$
t_{i}:=\left\{\operatorname{Id}, e^{1} e^{3}, e^{3}, e^{1}\right\},
$$

from which we obtain

$$
\bar{t}_{i}=\alpha_{-1}\left(t_{i}\right)=\left\{\operatorname{Id}, e^{3} e^{1},-e^{3},-e^{1}\right\} .
$$

Since no wedge product is involved, this can be used in the case $F^{\mu \nu} \neq 0$ also. Then one has to care, not to involve the common rules of reversion [21, 9, 25, 16].

Now we can build a base $P_{i j}$ of the matrix algebra, where every $P_{i j}$ has only one 1 at the $i$-th row and $j$-th column and the other elements zero

$$
P_{i j}:=\bar{t}_{i} P t_{j}
$$

Furthermore, we have

$$
\begin{aligned}
P_{i j} P_{k l} & =\delta_{j k} P_{i l} \\
\sum_{i=1}^{4} P_{i i} & =\mathbb{I} \\
P_{11}^{\sim} & =P_{11},
\end{aligned}
$$


if includes complex conjugation of the non-geometric factor $i$. One may notice, that the conjugation used in the Dirac adjoint is ${ }^{-}$and not ${ }^{\sim}$. The $i$ and $j$ are not an index, but a label of the base elements. We can construct a spinor base to represent the elements of $C L(\mathbb{R}, \eta)$. Therefore we set

$$
\begin{aligned}
\xi_{i} & :=P_{i 1}=\bar{t}_{i} P_{11} \\
\xi_{i}^{* T} & :=P_{1 i}=P_{11} t_{i} .
\end{aligned}
$$

Now, it is easily seen, that we have four possible choices to construct such spinor representations, due to the four idempotents $P_{i i}$, which yield identical matrix representations. The above choice of the $t_{i}$ and $\bar{t}_{i}$ ensures that the spinor representations of the $e^{\mu}$ equals the usual Dirac representation for vanishing $F^{\mu \nu}$. The fourfold possibility is well known, see Parra 26].

Due to the multiplication rule (53), left multiplication preserves this structure, while right multiplication does mix the four possibilities. In this way, it is not obvious, how one can connect ideal spinors and Dirac-Hestenes spinors (elements of $\left.C L^{+}\right)$, which are build up from all four spinor modules $\left(P_{i k}, k \in\{1,2,3,4\}\right.$, fixed) and are subjected to a right action, which indeed mixes these modules. The explanation of iso-spin from the right action of certain elements in the Dirac-Hestenes theory does thus involve up to four distinct spinor modules (particles).

The above developed method to start from the Grassmann algebra and the $j-$ sources give a unique construction to spinor modules. The ambiguity is thereby removed, which is of extreme importance in QFT, where the index-sets become infinite. Thus one has not to bother, which of the four (infinite many in QFT) modules to choose and how to establish their connection.

To establish this in Dirac theory, we have to translate equation (46) into our picture. One would expect from dimensional arguments, that the algebra $\Lambda\left[\mathbb{R}^{3}\right]$ should be large enough to carry the Dirac spinor (each of them has 8 real parameters). But, the mass term, with its unconvenient feature to mix even and odd parts of the state forces us to use $\Lambda\left[\mathbb{R}^{4}\right]$, with four sources $\left\{j_{1}, j_{2}, j_{3}, j_{4}\right\}$. A "state" $X$ is the written as $\left(\alpha_{i \ldots} \in \mathbb{C}\right.$, s.c. employed $)$

$$
X=\alpha_{0} \operatorname{Id}+\alpha_{i} j_{i}+\alpha_{i j, i<j} j_{i} \wedge j_{j}+\alpha_{i j k, i<j<k} j_{i} \wedge j_{j} \wedge j_{k}+\alpha_{i j k l, i<j<k<l} j_{i} . .
$$

The $\gamma$-matrices become via the definition (17 or 23) functions of $\partial, j$. Furthermore, we have to search for a "vacuum state", which has to fulfill the relation

$$
\partial^{x} \mid 0>_{F}=0 \quad \forall x \in \mathcal{V}(1,3) .
$$

We could achieve this by

$$
\mid 0>_{F}=\partial^{1} \partial^{2} \partial^{3} \partial^{4},
$$

but we prefer another choice, because we would like to require $\mid 0>_{F}$ to be a (primitive) idempotent element, which provides us an "scalar product". One should thus write $\left|0>_{F}<0\right|$ to emphasize this feature. Hence we define

$$
\left|0>_{F}<0\right|\left(\equiv \mid 0>_{F} \text { loosely }\right) \quad:=\partial^{1} \partial^{2} \partial^{3} \partial^{4} j_{4} j_{3} j_{2} j_{1} .
$$

This state obtains the properties

$$
\begin{aligned}
\left(\left|0>_{F}<0\right|\right)^{2} & =\left|0>_{F}<0\right| \\
X\left|0>_{F}<0\right| & =\left|X>_{F}<0\right| \\
\left|0>_{F}<0\right| X\left|0>_{F}<0\right| & =<X>\left|0>_{F}<0\right| .
\end{aligned}
$$

The functional picture, which is adapted to field theoretic considerations, has a very close connection to Crumeyrolle's construction of spinors [27. The distinction 
arises from the arbitrary bilinear form $B$ and from the fact, that the isotropic space was introduced only for technical reasons. The development here is not restricted to this special case.

We can now define a base, which is large enough to carry a representation of the $e^{\mu}$. This base is given as

$$
\mid \alpha>\in\left\{j_{\alpha}\left|0>_{F}<0\right|\right\},
$$

where $\alpha$ is an ordered possible empty index set out of $\{1,2,3,4\}$ and $j_{\emptyset} \equiv$ Id. If we represent the base elements on them self, we obtain a "spinor" representation

$$
\begin{aligned}
{\left[\left|0>_{F}<0\right|\right] } & =\left[\begin{array}{ccc}
1 & 0 & \cdots \\
0 & \ddots & \\
\vdots & &
\end{array}\right]_{16 \times 16} \\
{\left[j_{1}\left|0>_{F}<0\right|\right] } & =\left[\begin{array}{ccc}
0 & 0 & \cdots \\
1 & \ddots & \\
\vdots &
\end{array}\right]_{16 \times 16}
\end{aligned}
$$

etc. The other matrices are given by terms like

$$
\left[\left|0>_{F}<0\right| \partial^{1}\right]=\left[\begin{array}{ccc}
0 & 1 & \cdots \\
0 & \ddots & \\
\vdots & &
\end{array}\right]_{16 \times 16}
$$

which is a right action and thus not present in our formalism. The above given set of the $P_{i j}$, can thus be reobtained by identification of $i, j$ with $\alpha, \beta$ thus

$$
P_{i j} \approx j_{\alpha}\left|0>_{F}<0\right| \partial^{\beta} .
$$

The functional picture picks out one and only one of the spinor representations. The $\beta$-index in (46) is thus of the same kind as the above one. But due to the general construction including non-trivial $F^{\mu \nu}$ we need the full set of 16 elements and not 4 complex or 8 real ones. The representation matrices of the functional bases show no dependence of the metric $\eta^{\mu \nu}$ nor of the alternating part $F^{\mu \nu}$. This changes drastically if one calculates matrix representations of $e^{\mu}$ 's, which are highly asymmetric and $\eta^{\mu \nu}$ as $F^{\mu \nu}$ dependent [20]. $F^{\mu \nu}$ may be a function of space and time, even if one works within a static Minkowsky space ( $\eta^{\mu \nu} \equiv$ constant).

To compare Dirac theory with QFT, it is convenient to use Hamilton formalism. Formula (46) results in the algebraic picture as $\left(\gamma^{0-1}=\gamma^{0}\right)$

$$
i \frac{\partial}{\partial x^{0}} \psi=\sum_{k=1}^{3}\left(-\gamma^{0} \gamma^{k} \frac{\partial}{\partial x^{k}}-\frac{e}{\hbar c} \gamma^{0} \gamma^{k} A_{k}\right) \psi-\left(\frac{e}{\hbar c} A_{0}+m c \gamma^{0}\right) \psi .
$$

The transition into the functional picture is obtained with

$$
\begin{aligned}
\psi & \longrightarrow X\left|0>_{F}<0\right| \equiv \mid X> \\
\gamma^{\mu} & \longrightarrow \sum_{\nu}\left(B^{\mu \nu} j_{\nu}\right)+\partial^{\mu} .
\end{aligned}
$$

Hence we may calculate the functional energy equation

$$
E\left|X>=i \frac{\partial}{\partial x^{0}}\right| X>=H[j, \partial] \mid X>.
$$


The functional Hamiltonian $H[j, \partial]$ requires long winded calculations, which are not illuminating. But, comparing the structural form of this Dirac functional equation to functional equations of Dyson-Schwinger-Freese hierarchies in QFT [28] is quite interesting.

- The Hamilton formulation suggests to treat Dirac theory within Pauli algebra $\left(C L_{3,0}\right)$, which would reduce the dimension of the state space to 8 [6].

- The mass term, due to the $\gamma^{0}$ mixes odd and even parts of the state. This is a very uncommon feature in QFT, where the hierarchy equations decouple in odd and even ones. This is a kind of "super symmetry", which is known to be relevant in Dirac theory [30]. Therefore we identify the Yvon-Takabayasi angle as a measure of particle number non-conservation.

- The functional sources and states provide a metric and $F^{\mu \nu}$ independent set of base elements. In great contrast we observe a $\eta^{\mu \nu}$ and $F^{\mu \nu}$ dependence of the usual base elements $e^{\mu}$. Even in static Minkowsky space $\mathbf{M}_{1,3}$, there may be an up to now overlooked space-time dependence due to a nontrivial $F^{\mu \nu}=F\left(x^{\mu}, x^{\nu}\right)$. This plays an important role in QFT, where $F^{\mu \nu}$ can be identified with the propagator [9, 21].

- To cure the unconvenient features of functional Dirac theory, one should investigate from the beginning Dirac theory within $C L_{4,1}$, where also the nongeometric $i$ is turned into a geometric entity. The functional state space becomes then 32 dimensional, which equals the real degrees of freedom in complexified Dirac theory. Within this picture, there may be a chance to reobtain full QED, which is based on four Fock-like oscillator degrees of freedom, each described by ordinary four-component spinor field operators $\left(a_{\uparrow}^{\dagger}, a_{\downarrow}^{\dagger}, d_{\uparrow}, d_{\downarrow}\right)$. This may also be the link to the fourfold possibility obtained in Dirac-Hestenes theory by Parra.

- In usual Dirac theory, one postulates a priori the connection between the spinor and its adjoint. Due to this, the $F^{\mu \nu}$ is fixed (to zero). In our approach, the $F^{\mu \nu}$ is not fixed and has to be calculated from the theory. This requires a non-linear equation, which in QFT is obtained by the coupling to the vacuum.

- Because it is possible to calculate the functional Hamiltonian $H[j, \partial]$, one can ask for the "one-particle" theory, which results in this functional "field quantized" equation. This is the reversed question, how to quantize classical (spinor) fields in QFT. One obtains such an equation by "one-particle" projections $F<0 \mid \partial^{i}$

$$
\begin{aligned}
<0\left|\partial^{i} E\right| X> & =<0\left|\partial^{i} H[j, \partial]\right| X> \\
E \alpha^{i} & =H^{i} \alpha_{0}+\sum H^{i j} \alpha_{j}+\ldots
\end{aligned}
$$

This equations may be non-linear.

- Since in Dirac theory one does require the scalar product to be $\mathbb{C}$-valued, we have for one state

$$
<\Psi \mid \Psi>=\bar{\Psi}^{D} \Psi \quad \rightarrow \quad \mathbb{R} .
$$

Thus $\Psi$ has to be in the Lipschitz group $\Gamma(p, q)$, because only there we have

$$
\Phi_{\varepsilon}(\Psi, \Psi)=\alpha_{\varepsilon}(\Psi) \Psi \rightarrow \mathbb{R},
$$

and $\Psi$ is decomposable into one-vectors as selfadjoint to be $\mathbb{R}$ - and not $\mathbb{C}$ valued. This is not a usual requirement in QFT and thereby new. This 
picture turns the state $|\Psi\rangle$ to be in a Grassmannian. The classification of such manifolds is given in terms of Stiefel-Withney classes. An approach to the Dirac theory in such a tetrad formalism (mobiles of streamlines) is given by Krüger [29].

- From the features of the Dirac theory one may be able to construct adjoint (left) functional states, which then constitutes a functional metric (on elements of the Lipschitz group). This should be possible for non-linear theories (QCD, NLJ-models) also and is thus beyond the current QFT development and beyond current Fock space methods e.g. perturbation theory.

\section{Conclusion}

We developed a new method to study Dirac theory. This was motivated to search for an analogous picture, which was previously helpful in QFT. The Chevalley deformation provides a tool, to fix not only the quadratic form $\mathcal{Q}$ of the Clifford algebra in use, but also to fix the multi-vector structure. This multi-vector structure is needed to build scalar products and expectation values. The alternating (antisymmetric) part of the chosen bilinear form is thus an important part of the theory, even if it is usually absent.

The Dirac theory was shown to behave in an unexpected way if considered as a (toy) QFT. The mass term breaks the usual observed splitting of QFT functional equations in even and odd parts and breaks "particle number" conservation. This term is thus the source of a well known [30] super symmetry. The formulation of the theory suggests very strongly, that one should study Dirac theory by means of the Pauli algebra, see therefore Daviau [6], which is of course obscured by the mass term.

The possibility to have beside a constant Minkowsky metric a position dependent alternating part, opens new ways to study Dirac theory including vacuum effects.

We have shown, that the requirement to use "states" in QM involves new features, not observed in the operator formalism of Dirac-Hestenes or QF theory. In this sense does the Dirac-Hestenes theory bear no direct link to measurement since one does not explicit calculate (or fix by physical motivated assumptions) the $F^{\mu \nu}$ parameters, which therein are set implicitly zero.

\section{References}

[1] N. Bohr :On the construction of atoms and molecules Phil. Mag. 26 1-25 1913

[2] A. Sommerfeld : Münchner Ber. p. 4251915 and Zur Quantentheorie der Spektrallinien Ann. Phys. 51 1-94 1916

[3] W. Pauli :Zur Quantenmechanik des magnetischen Elektrons Z. Phys. 43 6016251927

[4] P.A.M. Dirac :The quantum theory of the electron (part I/II) Proc. R. Soc. London A117 610 1928, A118 3511928

[5] H. Krüger :New solutions of the Dirac equation for central fields in "The Electron" p. 49-81 (D. Hestenes, A. Weingartshofer eds., Kluwer Academic Publishers 1991)

[6] C. Daviau :Sur L'équation de Dirac dans l'alébre de Pauli (Ann. Fond. Louis de Broglie, to be published) 
[7] G. Lochak: Wave equation for a magnetic monopole Int. J. of Th. Phys. 2410 1985

[8] C. Daviau :Equation de Dirac non linéaire Thesis U Nantes 1993

[9] B. Fauser \& H. Stumpf :Positronium as an example of algebraic composite calculations in proceedings of the "International Conference on the Theory of the Electron (Cuautitlan 1995)" (J. Keller, Z. Oziewicz eds., 1996 in press) hep-th/9510193

[10] D. Hestenes :Observables, operators and complex numbers in the Dirac theory J. Math. Phys. 16(3) 5561975

[11] H. Grassmann (d.̈̈.) :Ausdehnungslehre Adolph Enslin/Berlin 1862

[12] Z. Oziewicz :From Clifford to Grassmann in "Clifford algebras and their applications in Mathematical Physics (Canterbury 1985)" (J.S.R. Chisholm, A.K. Common eds., Reidel/Dordrecht 1986)

[13] W. Greub :Multilinear algebra Springer/New York 1967

[14] W.A. Rodrigues, Q.A.G de Souza \& J. Vaz :Spinor fields and superfields as equivalence classes of exterior algebra fields pp. 177-198 in "Clifford algebras and spinor structures" (R. Ablamowicz, P. Lounesto eds. Kluwer 1995)

[15] C. Chevalley :The algebraic theory of spinors Columbia University Press/New York 1954

[16] R. Ablamowicz \& P. Lounesto :On Clifford algebras of a bilinear form with an antisymmetric part in "Clifford algebras with numeric and symbolic computations" p. 167-188 (R. Ablamowicz, P. Lounesto, J.M. Parra eds., Birkhäuser 1996)

[17] I.R. Porteous :Topological geometry Cambridge University Press 1981

[18] J.G. Maks :Modulo (1,1) periodicity of Clifford algebras and generalized (anti-) Möbius transformations Thesis TU Delft 1989

[19] P. Lounesto :Spinors and Brauer-Wall groups Report-HTKK-MAT-A124 1978

[20] B. Fauser : Vertex normalordering as a consequence of nonsymmetric bilinearforms in Clifford algebras J. Math. Phys. 37(1) 72-83 1996 (hep-th/9504055)

[21] B. Fauser :Clifford-algebraische Formulierung und Regularität in der Quantenfeldtheorie Thesis U Tübingen 1996

[22] R. Delanghe, F. Sommen \& V. Soucek :Clifford algebra and spinor valued functions, a function theory of the Dirac operator Kluwer 1992

[23] J.D. Bjorken \& S.D. Drell :Relativistische Quantenmechanik BI-Wissenschaftsverlag/Mannheim 1966

[24] D. Hestenes :"Space time algebra" Gordon and Beach 1966

P. Lounesto :Crumeyrolle's bivectors and spinors in "Clifford algebras and spinor structures" a special volume to the memory of Albert Crumeyrolle (R. Ablamowicz, P. Lounesto eds., Kluwer/Dordrecht 1995)

[25] B. Fauser :Clifford algebraic remark on the Mandelbrot set of two-component number systems Adv. in Appl. Clifford Alg. 6(1) 1996 in press (hep-th/9507133) 
[26] J.M. Parra: On Dirac and Darwin-Hestenes equation in "Clifford algebras and their application in mathematical physics (Montpellier 1989)" (A. Micali, R. Boudet, J. Helmstetter eds., Kluwer 1990)

[27] A. Crumeyrolle :Orthogonal and symplectic Clifford algebras, spinor structures Mathematics and its Applications, Kluwer 1990

[28] H. Stumpf \& Th. Borne :Composite particle dynamics in quantum field theory Vieweg/Braunschweig 1994

[29] H. Krüger :Classification of lightlike curves in spacetime under the group of $S O(1,3)$ motions in proceedings of the "International Conference on the Theory of the Electron (Cuautitlan 1995)" (J. Keller, Z. Oziewicz eds., 1996 in press)

[30] B. Thaler :The Dirac-equation Springer/Berlin 1992 